\title{
Physical investigations on the radiation damage of graphene oxide by IR pulsed laser
}

\author{
Letteria Silipigni $^{1 *}$, Lorenzo Torrisi ${ }^{1,2}$, Mariapompea Cutroneo $^{2,3}$ \\ ${ }^{1}$ Dipartimento di Scienze Matematiche e Informatiche, Scienze Fisiche e Scienze della Terra, MIFT, Università di Messina, V.le \\ F.Stagno d'Alcontres 31, 98166 S. Agata, Messina Italy \\ ${ }^{2}$ Dottorato di Ricerca in Fisica, MIFT, Università di Messina, Italy \\ ${ }^{3}$ Nuclear Physics Institute, CAS, 25068 Rez, Czech Republic
}

\begin{abstract}
Graphene oxide foils were irradiated by Nd:YAG laser at the moderated intensities of the order of $10^{8} \mathrm{~W} / \mathrm{cm}^{2}$ in vacuum. Measurements of atomic emission during the laser irradiation were performed with ion collectors and mass spectrometry, demonstrating a strong emission of carbon, oxygen and hydrogen atoms. Further investigations of the irradiated graphene oxide foils were carried out on pristine and laser irradiated samples by using Rutherford backscattering spectrometry and X-ray photoelectron spectroscopy. Results indicate that graphene oxide losses oxygen and hydrogen during the irradiation, changing its carbon content and its chemical and physical properties.
\end{abstract}

\section{Introduction}

Carbon has different crystallographic structures (allotropes), of which the most common ones are graphite and diamond.

Graphite has a three-dimensional layered structure in which tetravalent atoms of carbon are connected between them by three covalent bonds forming a hexagonal network structure of which any layer is called graphene. Different graphene layers are parallel between them. The bonds between layers are weak, thus the layers can slide easily over each other. The distance between layers is $0.335 \mathrm{~nm}$. Even if graphene only refers to an individual layer of graphite's planar honeycomb carbon lattice, the term graphene has also been adopted for up to 10 layers of carbon sheets by convention [1]. Graphene has peculiar physical and chemical properties, including excellent optical and mechanical properties, such as unprecedented highly versatile Young's modulus and tensile strength, high transmission over a wide wavelength range, high electrical and thermal conductivity, high biocompatibility, high surface effects with respect to volume ones [2].

Due to these properties the applications of graphene and its derivatives are wide range of; they can be employed as sensors, membranes, composite materials, biomaterials, in microelectronics, catalysis, etc.

Graphene oxide (GO) is defined as a monolayer of graphene with various oxygen-containing functional groups mainly in the form of hydroxyl $(\mathrm{C}-\mathrm{OH})$ and epoxy (C-O-C) groups attached on the basal plane with a very small contribution of carbonyl $(\mathrm{C}=\mathrm{O})$ and carboxyl $(\mathrm{O}-\mathrm{C}=\mathrm{O})$ groups which predominantly decorate the edges of the graphene sheets [3]. Therefore graphene oxide (GO) is a compound of carbon, oxygen, and hydrogen in variable ratios. It is generally obtained by treating graphite with strong oxidizers. Graphene oxide layers generally are about $1.1 \mathrm{~nm}$ thick; they are hydrophilic and easily hydrated when exposed to water vapor or immersed in liquid water. Graphene oxide decomposes when it is rapidly heated at moderately high temperatures $\left(\sim 300^{\circ} \mathrm{C}\right)$ or chemically treated with specific solvents, with formation of finely dispersed amorphous carbon and radicals [4]. The structure can be intercalated with many different atomic and molecular species conferring different properties to the GO layers. GO thin films, of the order of $100 \mathrm{~nm}$ in thickness, can be prepared to be near impermeable to gases, liquids or strong chemical agents.

Fig. 1 shows a typical graphene layer (a) and a graphene oxide layer (b) containing different functional groups.
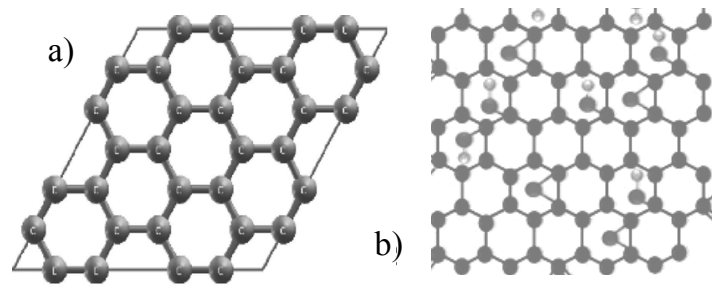

Fig. 1. Typical graphene layer (a) and graphene oxide layer containing different functional groups (b). 
The electrical conductivity of GO is strongly influenced by such functional groups and its band gap could be tuned from an insulator to a semimetal by the selective reduction of GO, where the term reduction is defined as a loss of oxygen. Generally GO has a low sheet resistance $(\sim 100-1000 \Omega$ per square), and a high optical transmittance $(\sim 80 \%$ at $550 \mathrm{~nm})$ [2].

Often structural defects are maintained low and their concentration depends on the synthesis process that is used to grow GO thin films [5].

In this paper the damage of GO due to fast and energetic laser pulses, of $3 \mathrm{~ns}$ and up to $500 \mathrm{~mJ} / \mathrm{cm}^{2}$, was investigated.

\section{Materials and Methods}

Graphene oxide foils were prepared by the graphite oxidation by using the Hummer's method as described in detail in the literature [6]. Thin GO foils, $7 \mu \mathrm{m}$ in thickness and $4 \mathrm{~cm}^{2}$ in surface, were employed in such investigation. Their synthesis was obtained at CANAM laboratory in Rez (Czech Republic).

An unfocalized Nd:YAG laser, at the $1064 \mathrm{~nm}$ wavelength, 3 ns pulse duration, $500 \mathrm{~mJ}$ maximum pulse energy, $1 \mathrm{~cm}^{2}$ spot size, was employed in the single pulse mode, up to an intensity of about $1.7 \times 10^{8} \mathrm{~W} / \mathrm{cm}^{2}$. This laser was used to irradiate GO foils in a vacuum chamber by using different laser pulse energies. $50 \mathrm{~mJ}$, $75 \mathrm{~mJ}, 100 \mathrm{~mJ}$, and single and double laser pulses, up to $500 \mathrm{~mJ}$ onto a surface of $1 \mathrm{~cm}^{2}$ were employed. The laser irradiation was monitored in-situ by using a Joulemeter for the laser pulse energy control. An ion collector (IC), connected in the time-of-flight (TOF) configuration to a fast oscilloscope for the measurements of the carbon and oxygen ion emission and a mass quadrupole spectrometer (MQS) for the gas degassing diagnostics were employed. A scheme of the used experimental set up is reported in Fig. 2.

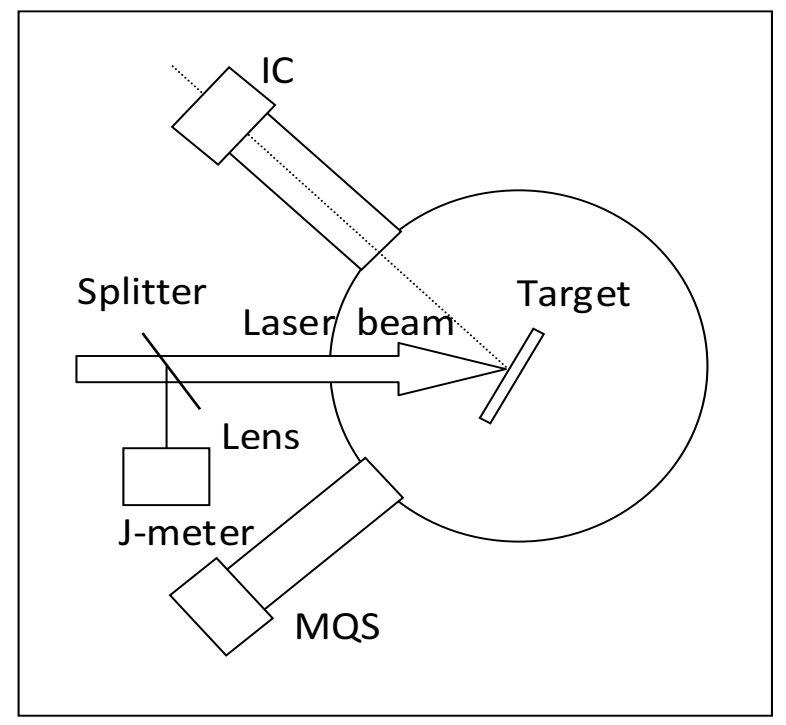

Fig. 2. Experimental set-up.

IC gives TOF spectra from which it is possible to evaluate the ion emission velocities and, as a first approximation, the charge states of the emitted ion species [7].

MQS provides spectra of the atomic and molecular species produced by the laser ablation of the sample surface; they were recorded from 1 up to $200 \mathrm{amu}$.

Rutherford backscattering spectrometry (RBS) analysis was performed by using a $2.0 \mathrm{MeV}$ helium beam in order to measure the composition and the stoichiometry of the sample before and after the laser irradiation [8].

X-ray photoemission spectroscopy (XPS) was employed to analyze the structure of the graphene oxide foils before and after the laser irradiation. It is a surfacesensitive analytical technique that is useful to determine the chemical environment of atoms, in this case GO carbon atoms. XPS analysis was carried out on a VG Scientific system operating, under $3 \times 10^{-8}$ mbar, to an anodic voltage of $10 \mathrm{kV}$ in the constant pass-energy mode at $20 \mathrm{eV}$ with an energy resolution [full width at half maximum (FWHM)] of $1.0 \mathrm{eV}$ as detected by the Ag $3 \mathrm{~d}_{5 / 2}$ emission. The spectrometer used a nonmonochromatic Al K $\alpha$ X-ray source $(h v=1486.6 \mathrm{eV})$ operating at $320 \mathrm{~W}$. The channeltron voltage was fixed at $2.5 \mathrm{kV}$ during each run. Owing to sample charging, the carbon $\mathrm{C} 1 s$ line at $285.0 \mathrm{eV}$ was used as reference for the determination of the binding energy position of all the investigated core levels. A Shirley background function was utilized to subtract the inelastic background at all the XPS spectra.

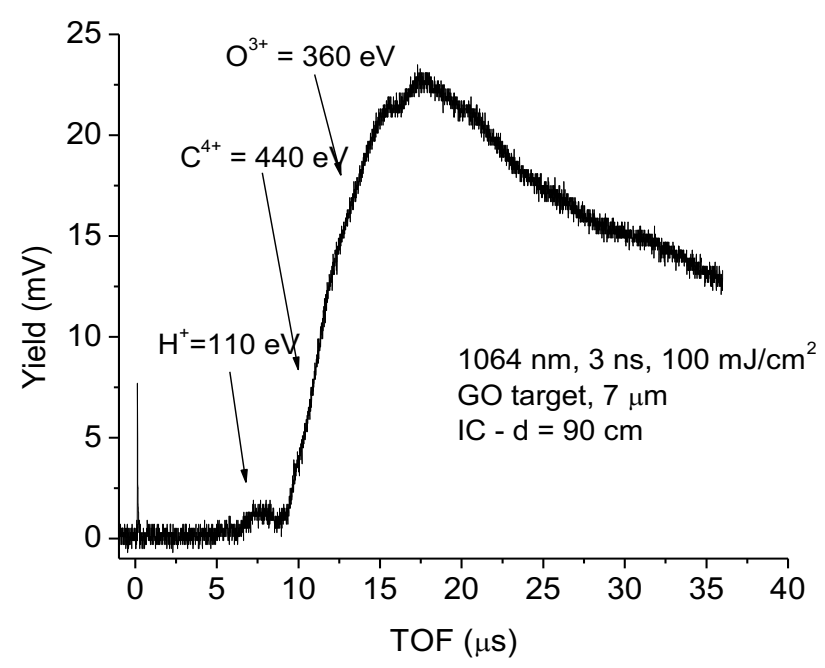

Fig. 3. IC-TOF spectrum of ions emitted during the GO laser irradiation.

\section{Results}

The laser irradiation of GO generates a plasma in front of the target emitting visible light, UV, soft X-rays, electrons and ions, as reported in previous investigations [9]. The different electron and ion mobility produces a non equilibrium plasma accelerating ions along the normal to the target surface. 
Fig. 3 shows a typical IC-TOF spectrum recorded during a laser shot irradiation of $\mathrm{GO}$ placed at $90 \mathrm{~cm}$ distance from the IC detector. The spectrum shows the emission of protons, carbon and oxygen ions. The protons are detected at a kinetic energy of $110 \mathrm{eV}$ : on the basis of the Coulomb Boltzmann Shifted (CBS) distribution, it means that the acceleration is of $110 \mathrm{eV}$ per charge state. The carbon and oxygen ions are detected at a maximum kinetic energy of $440 \mathrm{eV}$ and 360 $\mathrm{eV}$, respectively. Thus, measurements indicate a maximum charge state of $4+$ and $3+$ for the carbon and oxygen ions, respectively. Such charge states indicate that plasma electrons have energy up to the potential ionization energy to obtain $\mathrm{C}^{4+}$ and $\mathrm{O}^{3+}$, which correspond to $64 \mathrm{eV}$ and $55 \mathrm{eV}$, respectively. Because the ionization potential of $\mathrm{O}^{4+}$ is $77 \mathrm{eV}$, the maximum electron energy should be lower than this value. Assuming the maximum electron energy to be about 65 $\mathrm{eV}$ and the mean energy of the Boltzmann distribution to be a third of this maximum value, the electron mean energy of the plasma is of about $21 \mathrm{eV}$. Thus the mean electron plasma temperature $(k T=2 E / 3)$ corresponds to about $14 \mathrm{eV}$.

During the plasma formation the MQS spectrometer detects the emitted atoms and molecular species. Our analysis was performed by fixing some masses of interest, such as 1,12 and $16 \mathrm{amu}$, corresponding to the atomic presence of $\mathrm{H}, \mathrm{C}$ and $\mathrm{O}$, respectively.

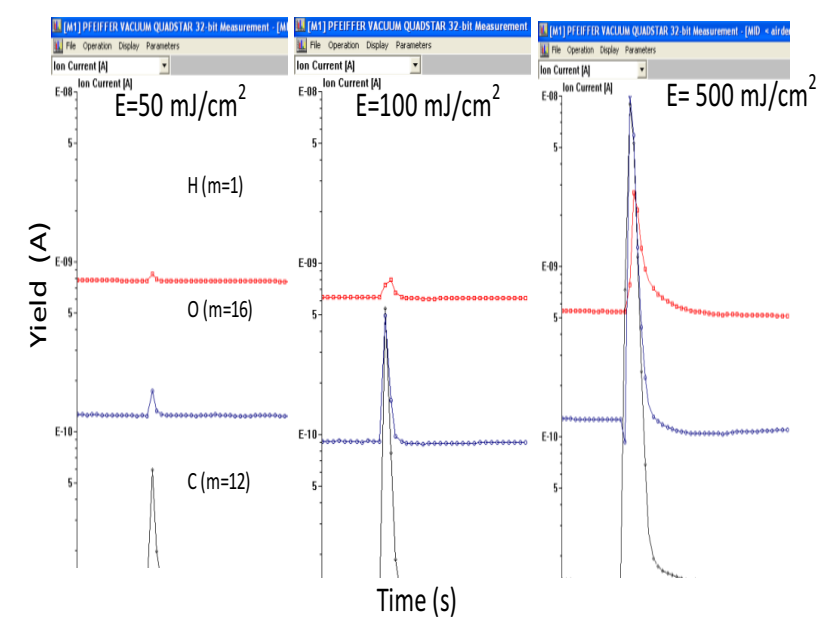

Fig. 4. Typical MQS spectra of emitted $\mathrm{C}, \mathrm{O}$ and $\mathrm{H}$ as a function of the time and of the laser pulse energy density.

Fig. 4 reports three typical MQS spectra of the emitted species $(\mathrm{H}, \mathrm{O}$ and $\mathrm{C})$ as a function of different laser pulse energy density, from $50 \mathrm{~mJ} / \mathrm{cm}^{2}$ up to $500 \mathrm{~mJ} / \mathrm{cm}^{2}$. Such fixed masses were plotted as a function of the time in condition of laser switched off and laser switched on, in order to evince their net yield during the laser irradiation. Spectra indicate that the GO emits carbon, oxygen and hydrogen, and other molecular groups containing oxygen, proportionally to the laser released energy. Results show that the laser ablation produces a strong emission of hydrogen, carbon and oxygen, reducing the content of oxygen and increasing the residual carbon content of the sample.

MQS yields are proportional to the GO composition, which was controlled by using a $2.0 \mathrm{MeV}$ helium RBS analysis. These spectra have evinced a composition in which oxygen decreases significantly with the laser irradiation, proportionally to the absorbed energy. Fig. 5 reports two typical RBS spectra relative to the analysis of the pristine graphene oxide (a) and the laser reduced graphene oxide (b).

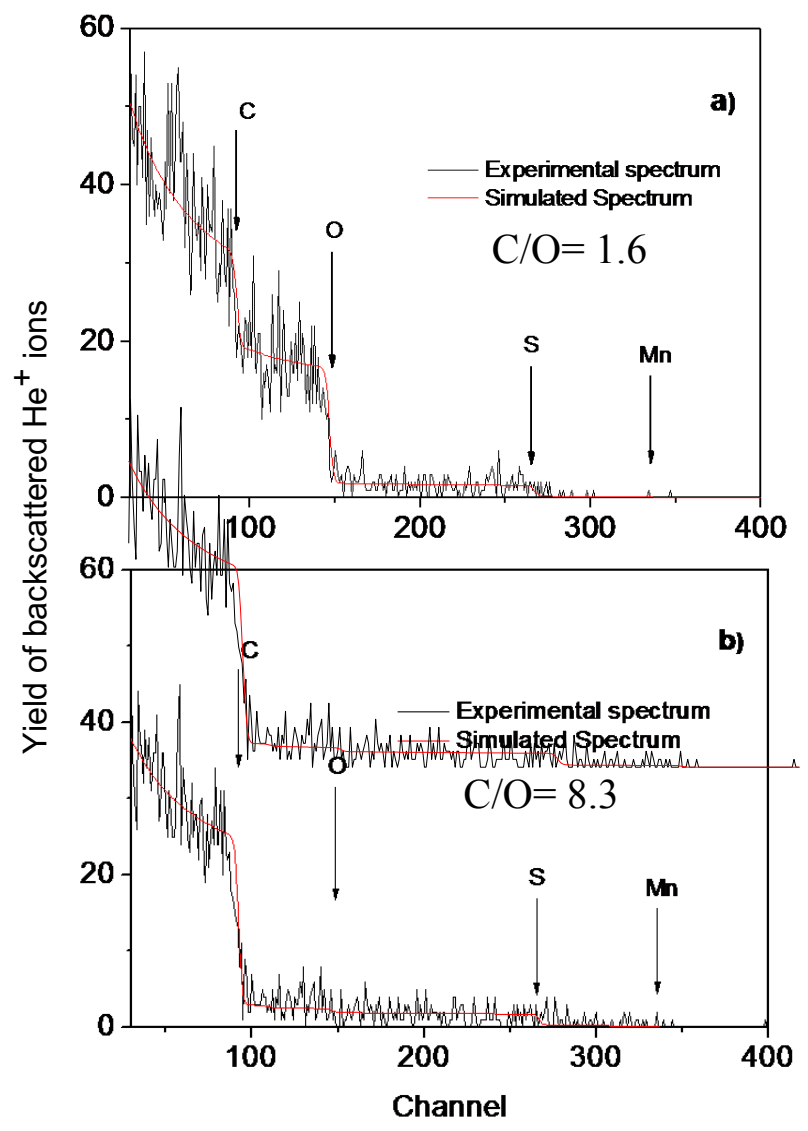

Fig. 5. RBS spectra relative to the pristine GO (a) and to the laser irradiated GO (b).

The first spectrum is rich in oxygen, while in the second one there is a strong oxygen reduction. The continuum lines represent the simulated spectra by using SIMNRA code [10]. Moreover, S and Mn elements are present as contaminants in the pristine sample. The quantitative analysis has demonstrated that the carbon to oxygen atomic ratio, $\mathrm{C} / \mathrm{O}$, is 1.6 in the pristine $\mathrm{GO}$ sample and it increases to 8.3 in the laser irradiated GO sample by using a laser pulse energy density of about $400 \mathrm{~mJ} / \mathrm{cm}^{2}$.

XPS analyses were performed in the pristine and laser irradiated GO foils, in order to investigate about the chemical bonds of carbon with the different functional groups present in the graphene oxide structure as a function of the laser energy density irradiation. Fig. 6 shows the comparison between the XPS spectra, performed in the $\mathrm{C} 1 \mathrm{~s}$ and $\mathrm{O} 1 \mathrm{~s}$ core level regions for the pristine $(a)$ and laser irradiated $\mathrm{GO}(b, c)$ foils in order to evince the carbon and the oxygen content and their 
chemical groups, respectively. The laser treated GO foils were irradiated at $50 \mathrm{~mJ} / \mathrm{cm}^{2}(b)$ and at $200 \mathrm{~mJ} / \mathrm{cm}^{2}(c)$ energy densities respectively.
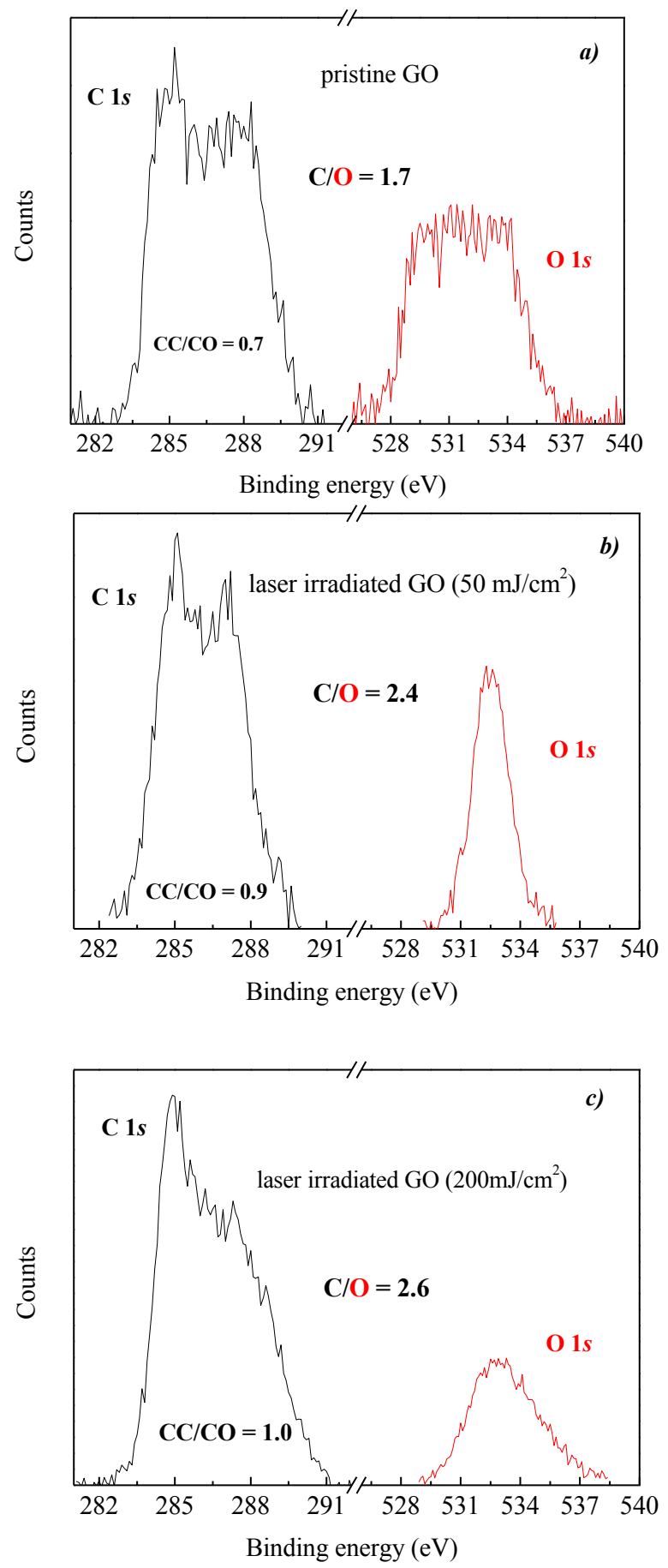

Fig. 6. Comparison of the $\mathrm{C} 1 \mathrm{~s}$ and $\mathrm{O} 1 \mathrm{~s}$ core level XPS spectra revealed for the pristine $\mathrm{GO}(a)$ and laser irradiated GO foils at $50 \mathrm{~mJ} / \mathrm{cm}^{2}(b)$ and $200 \mathrm{~mJ} / \mathrm{cm}^{2}(c)$.

The untreated GO foil high-resolution C1s XPS spectrum (see fig. $6 a$ ) consists of a peak at about 285.0 $\mathrm{eV}$, followed by a second large structure at about 288.0 $\mathrm{eV}$ that can be fit to peaks at about $286.8 \mathrm{eV}$ and at
$288.4 \mathrm{eV}$. These features can be attributed to $\mathrm{C}-\mathrm{C}$ and $\mathrm{C}=\mathrm{C}$ bonds (which cannot be detected separately due to the XPS spectrometer resolution), $\mathrm{C}-\mathrm{O}$ (in epoxy and hydroxyl groups) and $\mathrm{C}=\mathrm{O}$ (in carbonyl groups) links, respectively [11].

In the following $\mathrm{CC}$ refers to the sum of the $\mathrm{C}-\mathrm{C}$ and $\mathrm{C}=\mathrm{C}$ bonds, while $\mathrm{CO}$ designates all combinations of links between $\mathrm{C}$ and $\mathrm{O}$ atoms.

When GO is laser irradiated the ratio of the different peaks changes. In particular at $50 \mathrm{~mJ} / \mathrm{cm}^{2}$ (see Fig. $6 b$ ) the $285.0 \mathrm{eV}$ peak, due to the $\mathrm{C}-\mathrm{C}$ and $\mathrm{C}=\mathrm{C}$ links, and the $\mathrm{C}-\mathrm{O}$ peak at about $287.1 \mathrm{eV}$ increase as well the $\mathrm{C}=\mathrm{O}$ peak at about $289.2 \mathrm{eV}$ decreases; at 200 $\mathrm{mJ}$ (see Fig. $6 c$ ) the 285.0 peak continues to grow while the $\mathrm{C}-\mathrm{O}$ and $\mathrm{C}=\mathrm{O}$ peaks at about $287.1 \mathrm{eV}$ and $288.8 \mathrm{eV}$ respectively decrease. Consequently, in going from the pristine GO to laser irradiated GO $\left(200 \mathrm{~mJ} / \mathrm{cm}^{2}\right)$, the ratio between the areas of the $\mathrm{CC}$ and $\mathrm{CO}$ peaks increases from 0.7 to 1.0 , indicating a partial removal of oxygen-containing functional groups and, therefore, a partial reduction of GO in good agreement with RBS and MQS data.

As regards the O1s XPS spectra, initially the O1s spectrum indicates the presence of two large peaks at about $530.0 \mathrm{eV}$ and $533.3 \mathrm{eV}$ (see Fig. $6 a$ ), due to $\mathrm{C}=\mathrm{O}$ and $\mathrm{C}-\mathrm{OH}$ groups respectively, according to the literature [12]. After the $50 \mathrm{~mJ}$ laser irradiation, the intensity of the peak at lower binding energies decreases (see Fig. 6b) up to disappear at $200 \mathrm{~mJ}$ (see Fig. 6c), while at this laser energy density the presence of the only peak at about $533.1 \mathrm{eV}$ indicates that $\mathrm{C}-\mathrm{OH}$ groups are still present and are difficult to further reduce in good agreement with the literature [12]. The atomic ratio of carbon to oxygen (the area of the $\mathrm{C} 1 \mathrm{~s}$ peak corrected for its tabulated atomic sensitivity factor divided by the area of the O1s peak corrected for its tabulated atomic sensitivity factor) was calculated after each treatment and the obtained results are shown in Table I.

Table I. The values of the $\mathrm{C} 1 \mathrm{~s} / \mathrm{O} 1 \mathrm{~s}$ atomic concentration ratio obtained by high resolution XPS spectra.

\begin{tabular}{l|c}
\hline & $\mathrm{C} 1 s / \mathrm{O} 1 s$ atomic ratio \\
\hline Untreated GO foil & 1.7 \\
\hline $50 \mathrm{~mJ}$ laser irradiated GO foil & 2.4 \\
\hline $200 \mathrm{~mJ}$ laser irradiated GO foil & 2.6 \\
\hline
\end{tabular}

The XPS spectra deduced $\mathrm{C} / \mathrm{O}$ atomic ratio value, reported in Table I for the pristine GO foils is in agreement with that derived by the RBS spectra. More detailed investigations will be carried out shortly to shed light on the matter.

\section{Discussion and conclusions}

As reported in the literature, a key topic in the research and applications of GO is its reduction, which partly restores the structure and properties of graphene.

In this work, we have demonstrated that the pulsed laser beam can be used to modify the chemical and 
physical properties of $\mathrm{GO}$ by reducing its content in oxygen and increasing the one in carbon.

A strong emission of carbon, oxygen, hydrogen atoms is produced by the laser irradiation as indicated by the IC-TOF and MQS spectra.

By means of the RBS and XPS techniques, we have observed that the laser treatment at different laser pulse energy densities generates $\mathrm{GO}$ with defined $\mathrm{C} / \mathrm{O}$ atomic ratios and specific oxygen functionalities.

In particular, the analysis of the XPS spectra of the $\mathrm{C} 1 \mathrm{~s}$ and $\mathrm{O} 1 \mathrm{~s}$ core levels indicate that a loss of some oxygen functional groups occurs in GO upon laser irradiation. This loss increases as laser density energy grows and it has been confirmed by the RBS data.

Reported measurements are in agreement with the literature describing similar studies performed by using ion beam lithography of GO transforming the insulator pristine GO in the final conductive rich carbon graphene [13].

Our results indicate that pulsed laser irradiation is a good tool to reduce GO and it has important advantages over conventional chemical or thermal treatments since it is rapid and it can be applied directly to the GO film deposited, not requiring dangerous or toxic reagents.

However, the oxidation/reduction control by means of the laser irradiation must be further investigated in details to better understand how to tune the band gap in GO by varying the oxygen concentration as a function of the laser parameters (wavelength, pulse energy, pulse duration,...). For this purpose a more detailed XPS analysis will be undertaken together with an accurate Raman investigation. A deeper understanding of the oxidation and reduction processes will allow us to better control the attaching and elimination of some functional groups to some specific locations on the carbon plane in order to realize innovative devices in various fields ranging from electronics, energetics to optoelectronics.

Authors thank the CANAM (Center of Accelerators and Nuclear Analytical Methods) infrastructure (LM2015056). This work was supported by the "Research and Mobility" project of Messina University No. 74893496, scientifically coordinated by Professor L. Torrisi.

\section{References}

1. M. Aliofkhazraei, N. Ali, W.I. Milne, C.S. Ozkan, S. Mitura, J.L. Gervasoni (eds.), Graphene science handbook-Mechanical and chemical properties, CRC Press, T\&F Group, Boca Raton (2016).

2. M. Aliofkhazraei, N. Ali, W.I. Milne, C.S. Ozkan, S. Mitura, J.L. Gervasoni (eds.), Graphene science handbook- Electrical and Optical properties, CRC Press, T\&F Group, Boca Raton (2016).

3. D.R. Dreyer, S. Park, C.W. Bielawski and R.S. Ruoff, Chem.Soc.Rev. 39, 228-240 (2010).

4. Z. Wang, D. Xu, Y. Huang, Z. Wu, L. Wang and

X. Zhang, Chem. Commun. 48, 976-978 (2012)
5. C. Gomez-Navarro, J.C. Meyer, R.S. Sundaram, A. Chuvilin, S. Kurasch, M. Burghard, K. Kern, U. Kaiser,Nano Lett. 10, 1144-1148 (2010).

6. J. Chen, B. Yao, C. Li, G. Shi, Carbon 64, 225-229 (2013)

7. L. Torrisi, G. Ceccio, N. Restuccia, E. Messina, P. G. Gucciardi, M. Cutroneo, Laser and Particle Beams 35(2), 294-303 (2017)

8. W.K. Chu, J.W. Mayer and M.A. Nicolet (eds.), Backscattering Spectrometry, Academic Press, New York, (1978)

9. L Torrisi, Nukleonika, 60 (2), 207-212 (2015)

10. SIMNRA Code, actual website 2017: http://home.mpcdf.mpg.de/ mam/

11. G. Sobon, J. Sotor, J. Jagiello, R. Kozinski, M. Zdrojek, M. Holdynski, P. Paletko, J. Boguslawski, L. Lipinska, and K.M. Abramski, Opt. Express 20, 19463- 19473 (2012).

12. D. Yang, A. Velamakanni, G.Bozoklu, S. Park, M. Stoller, R.D. Piner, S.Stankovich, I. Jung, D.A.Field, C.A.Ventrice Jr, R.S.Ruoff, Carbon 47,145-152 (2009)

13. D. E. Lobo, J. Fu, T. Gengenbach, and M. Majumder, Langmuir 28, 14815-14821 (2012)p 